\title{
POWER-EFFICIENT LOW-STRAY FIELD HYBRID MAGNETS FOR MAGLEV TECHNOLOGY
}

\author{
Daria Arslanova \\ JSC "NIIEFA", Russia \\ arslanova@sintez.niiefa.spb.ru
}

\author{
Alexey Firsov \\ JSC "NIIEFA", Russia \\ firsov@sintez.niiefa.spb.ru
}

\author{
Vladimir Kukhtin \\ JSC "NIIEFA", Russia \\ kukhtin@sintez.niiefa.spb.ru
}

\author{
Eugeny Lamzin \\ JSC "NIIEFA", Russia \\ lamzin@sintez.niiefa.spb.ru
}

\author{
Mikhail Larionov \\ JSC "NIIEFA", Russia \\ larionov@sintez.niiefa.spb.ru
}

\author{
Andrey Nezhentzev \\ JSC "NIIEFA", Russia \\ nezhencev@sintez.niiefa.spb.ru
}

\author{
Dmitri Ovsyannikov \\ St. Petersburg State University,, Russia \\ dovs45@mail.ru
}

\author{
Igor Rodin \\ JSC "NIIEFA", Russia \\ rodin@sintez.niiefa.spb.ru
}

\author{
Nikolay Shatil \\ JSC "NIIEFA", Russia \\ shatil@sintez.niiefa.spb.ru
}

\author{
Sergey Sytchevsky \\ St. Petersburg State University, Russia \\ sytch-sie@yandex.ru
}

\author{
Vyacheslav Vasiliev \\ JSC "NIIEFA", Russia \\ vvnm@mail.ru
}

\author{
Anatoly Zaitsev \\ St. Petersburg Transport University, Russia \\ nozpgups@mail.com
}

Article history:

Received 02.07.2021, Accepted 25.11.2021

\begin{abstract}
A hybrid suspension system is proposed for maglev transport that utilizes electromagnets (EM) in combination with permanent magnets (PM). Several design schemes are compared searching for optimum performance. Sufficient reduction of power consumption and stray field is achieved on the hybrid configuration as compared to conventional EM suspension systems.
\end{abstract}

\section{Key words}

magnetic levitation, vehicle, electromagnetic suspension, magnetic field, permanent magnet

\section{Introduction}

The maglev transportation technology utilizes magnetic suspension to provide propulsion of a levitated vehicle along the track without mechanical contact. The vehicle is suspended with the force produced by electromagnets or permanent magnets [Bocharov, Nagorsky, 1991; Dzenzersky et.al., 2001; Kim, 2007; Zaitzev et.al, 2010]. This eliminates contact friction, and the train motion is only affected by air and magnetic drag. A linear motor can propel a maglev vehicle with the speed much higher than achievable for conventional wheel-rail trains.
Design schemes for the electromagnetic suspension (EMS) are discussed. EMS uses an attraction force generated by interaction of the electromagnets on the vehicle and ferromagnetic guideways. A constant air gap is kept between the suspended vehicle and the track. The gravitational force acting on the vehicle is equal to the attraction force that depends on the current of EM coils and the size of the levitation gap. The EMS system is inherently unstable, and the active suspension control must be applied to ensure the constant levitation gap and the train stability. The control strategy is in general relies on the continuous and fast adjustment of the EM coil current [Zhuravlyov, 2003; Balandin, Kogan, Biryukov, 2017].

The EMS levitation is cost- and power-consuming as the attraction force is proportional to the coil current. In addition to heavy power sources, cooling systems are required to prevent overheating of the electromagnets due to high currents.

To reduce power consumption, a hybrid suspension is utilized with a combination of EM coils and permanent magnets [Amoskov et.al., 2018a; Andreev et.al., 2019]. The force generated by the permanent magnet adds to the electromagnetic attraction, and therefore reduces the coil current required to suspend the vehicle. The hybrid 

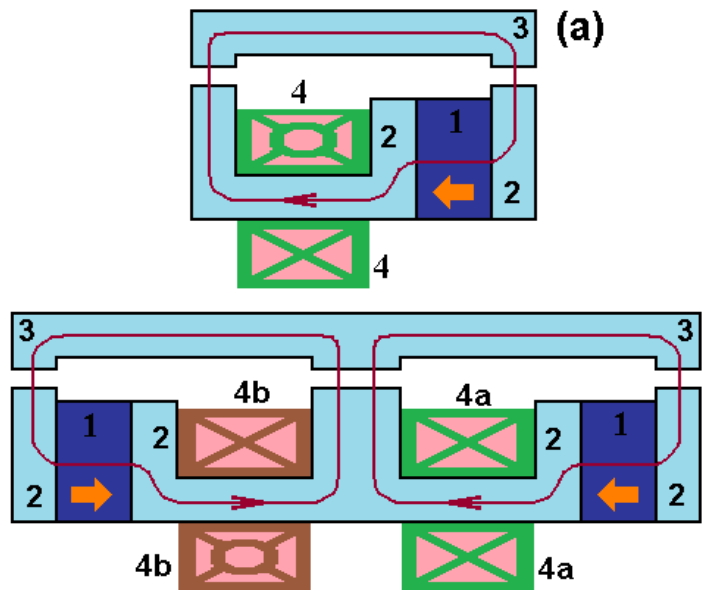

(b)

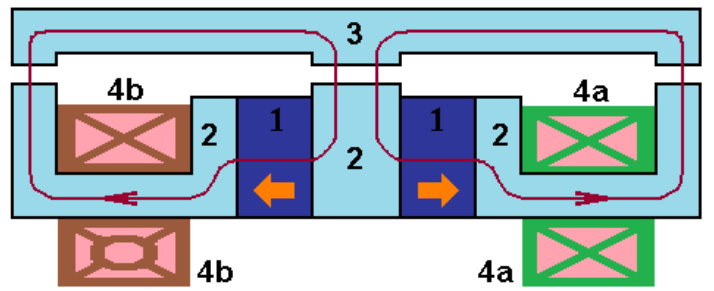

(c)

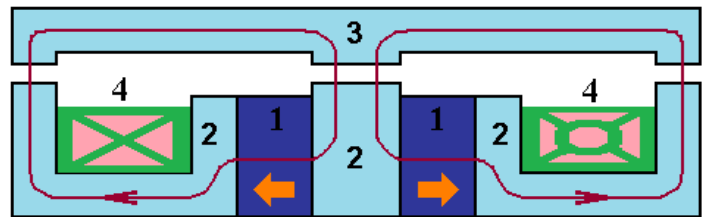

(d)

Figure 2. Modified designs of hybrid PM-EM system shown in Fig.1e: (a) - original configuration, (b, doubled magnet configurations; (d) single-coil configuration. Notation: (1) - PM, (2) - magnetic core, (3) - ferromagnetic guideway. (4) - EM.
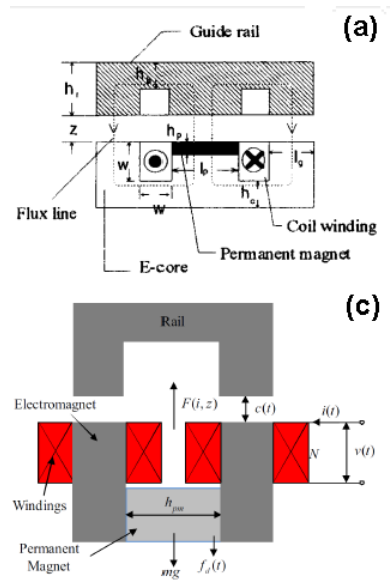

(e)

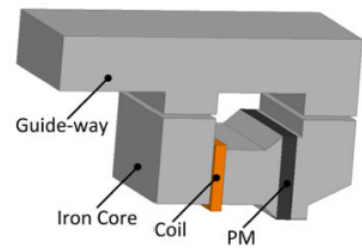

(a)

(c)
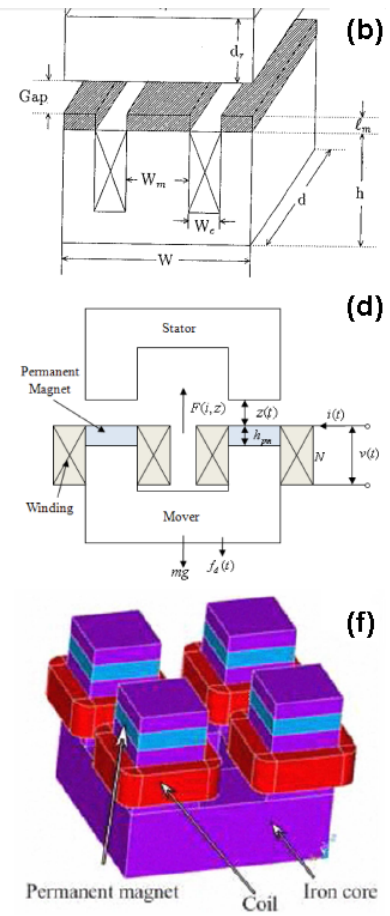

(d)

(f)
Figure 1. Hybrid suspension systems: [Tzeng and Wang, 1994] (a), [Onuki and Toda, 1993] (b), [Kim et.al., 2013] (c), [Kim et.al., 2010] (d), [Safaei et.al., 2015] (e), [Erkan et.al., 2011] (f). suspension makes it possible to increase payload without increase in power consumption.

To ensure a constant air gap between the vehicle and the track at varying load, a change of attraction force generated by PM can be employed. A possible solution is described in the US patent [Patent 7533616]. The Russian patent [Patent 2573524] proposes to adjust the air gap with a pair of electromagnets located above and below the ferromagnetic guideway.

Both conventional EMS and hybrid PM-EMS enable two principal functionalities: (1) suspension owing to attraction force between the magnet and ferromagnetic guideways and (2) dynamic adjustment of the air gap. The suspension control algorithm utilizes a feedback of the EM coil current. A rated coil current should provide suspension of the load with the desired air gap. The current deviations are regulated so that to keep the air gap constant.

Typically, the rated current in a conventional EMS is ten(s) times higher than deviation amplitudes. This implies high power consumption. In a hybrid suspension (e.g. [Patent 2611858]), the rated current is comparable to an average current deviation that results in low-power performance.

\section{Hybrid suspension}

There are various configurations to add PM in the magetic circuit of a hybrid suspension. Fig.1 illustrates several implementations.

High-coercivity PM (NdFeB) provides main load capacity, while EM coils are used for dynamic control and levitation stability. The attraction force generated by PM neutralizes the gravitational force and enables levitation of the vehicle with a certain air gap. With variable load during loading or unloading only a low coil current is used to adjust the air gap. The Ohmic power consumption is minimized as the load is levitated even at zero current in the EM coils. This is called the zero-power control [Morishita et.al., 1989]. Another advantage of the hybrid suspension is its lower size and weight as compared to EMS [Wang and Tzeng, 1994].

The hybrid suspensions presented in Figs.1c and 1d are most studied. One of the latest design solutions [Safaei et.al., 2015] shown in Fig.1e offers lower energy cost and higher lifting force. However, this configuration suffers from a rather high stray field outside the air gap between the PM polar area and the ferromagnetic guideways. This may lead to sticking of foreign objects and worsened maintainability, electromagnetic compatibility, and environmental safety.

A new design concept with improved performance is considered in this paper. The hybrid suspension from 


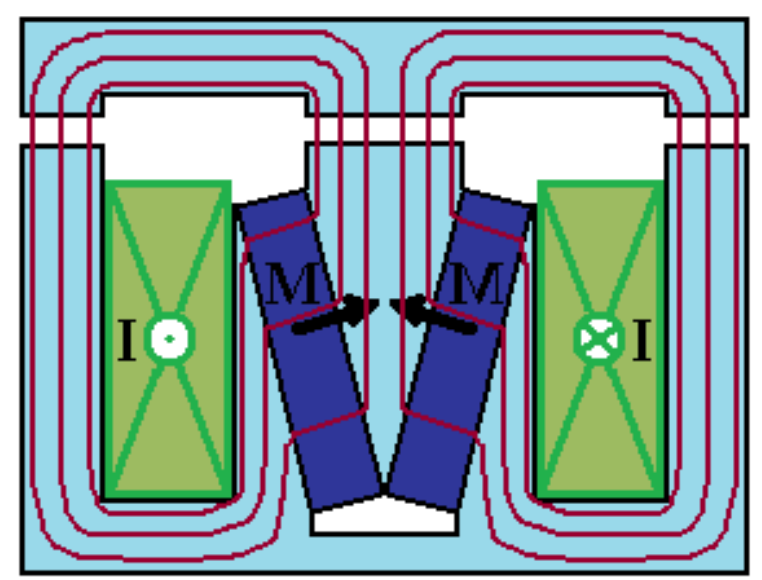

Figure 4. Hybrid suspension with V-shaped PM.


Figure 3. Magnetic flux in hybrid suspension system: hybrid PMEM system shown in Fig.2a (a) modified PM-EM system shown in Fig 2d (b). Notation: (1) - PM, (2) - magnetic core, (3) - ferromagnetic guideway, (4) - EM, (5) - stray field.

Fig.1e has been modified as shown in Fig.2. Fig.2a is the original magnetic scheme. The magnetic flux direction is indicated with arrows. Figs.2b,c present two configurations employing a pair of the Fig.2a magnets in different arrangements. The doubled magnets provide better concentration of the magnetic flux at the middle pole, and the total magnetic force rises more than twice. This yields to a $20-30 \%$ increase in the specific magnetic force as compared to the original configuration. The doubled configurations, Fig.2c in the first place, also provide substantial decrease of magnetic moment due to subtractive polarity of PMs, thus resulting in low stray field. The scheme in Fig.2d utilizes a single horizontal EM coil instead of the vertical coil used in the original configuration. This reduces the conductor mass by twofold while the magnetic characteristics remain unchanged. The single-coil configuration has been patented [Patent 2739939].

\section{Hybrid suspension with V-shaped PM}

The modified hybrid suspension [Patent 2739939] has the same drawback as the original scheme [Safaei et.al., 2015]. Stray field outside the working zone reaches up to $15-20 \%$ of the generated magnetic flux as illustrated in Fig. 3 In the plot pale areas indicate stray field associated with lower sides of PM.

The concept proposed in [Patent 2743753] enables sufficient improvement of the field distribution. This configuration employs a V-shaped PM assembly as shown in Fig.4. The permanent magnets are tilted symmetrically around the vertical axis. The magnetic circuit has a horizontal bridge at its lower side near the V-joint. The bridge is separated with an air gap from the joint thus preventing the magnetic flux closure. Such configuration ensures high mechanical strength and low stray field without deteriorating dynamic performance. For comparison, a similar bridge introduced in the single-coil structure (Fig.2d) would bypass the generated magnetic flux almost entirely so that the levitation force falls to zero.

Performance of the V-shaped hybrid system has been studied in a series of parametric simulations with detailed numerical models. Well-proven codes created for EM computations of accelerators and tokamak magnets were adapted to the simulations. The applied software tools are protected by national patents and certificates [Amoskov et.al., 2014a; Amoskov et.al., 2015a; Amoskov et.al., 2015b; Amoskov et.al., 2016; Amoskov et.al., 2018b; Amoskov et.al., 2014b; Amoskov et.al., 2008]. Virtual prototyping was supported by measurements on a test setup to validate the models and optimize the hybrid suspension design.

Figs.5 and 6 present a simulated field map of the Vshaped configuration in a comparison with the field from the vertical PM. The plots demonstrate that the V-shaped PM design is capable to reduce sufficiently stray field of the hybrid suspension system.

As compared to the previous configurations (Figs.2a and 2d), the hybrid suspension with V-shaped PM has improved suspension force characteristics. The force characteristics of a suspension system may be assessed through the parameter $k$ :

$$
k=(F \times h) / P,
$$

where $F[\mathrm{~N}]$ is the attraction force generated by $\mathrm{PM}$ at the air gap $h$ and zero current in EM coils; $P[\mathrm{~N}]$ is the weight of the suspension system.

$k$ reflects the magnetic circuit efficiency and rises with reduction in the weight. The proposed hybrid suspension system has $k$ twice higher than that of previous configurations, therefore offering an advantage of a lesser mass. 


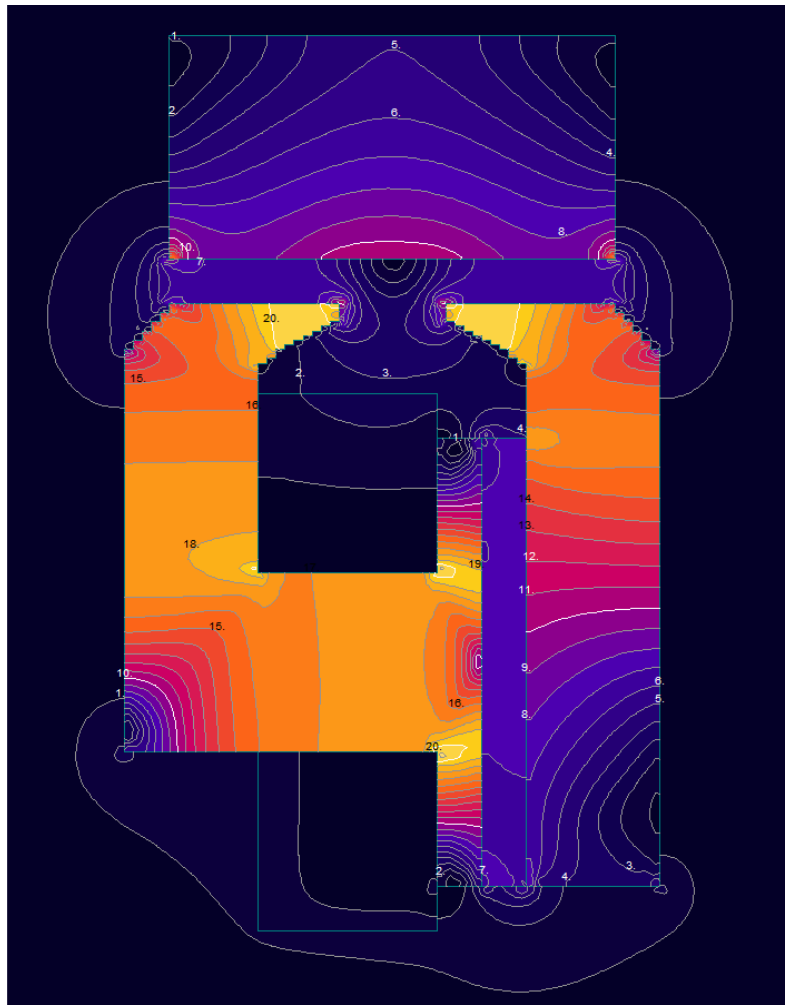

Figure 5. Simulated field map for hybrid suspension system with vertical arrangement of PM (Fig. 3b). Coil current $I=4 \mathrm{kA}$, air gap $h=5 \mathrm{~mm}$

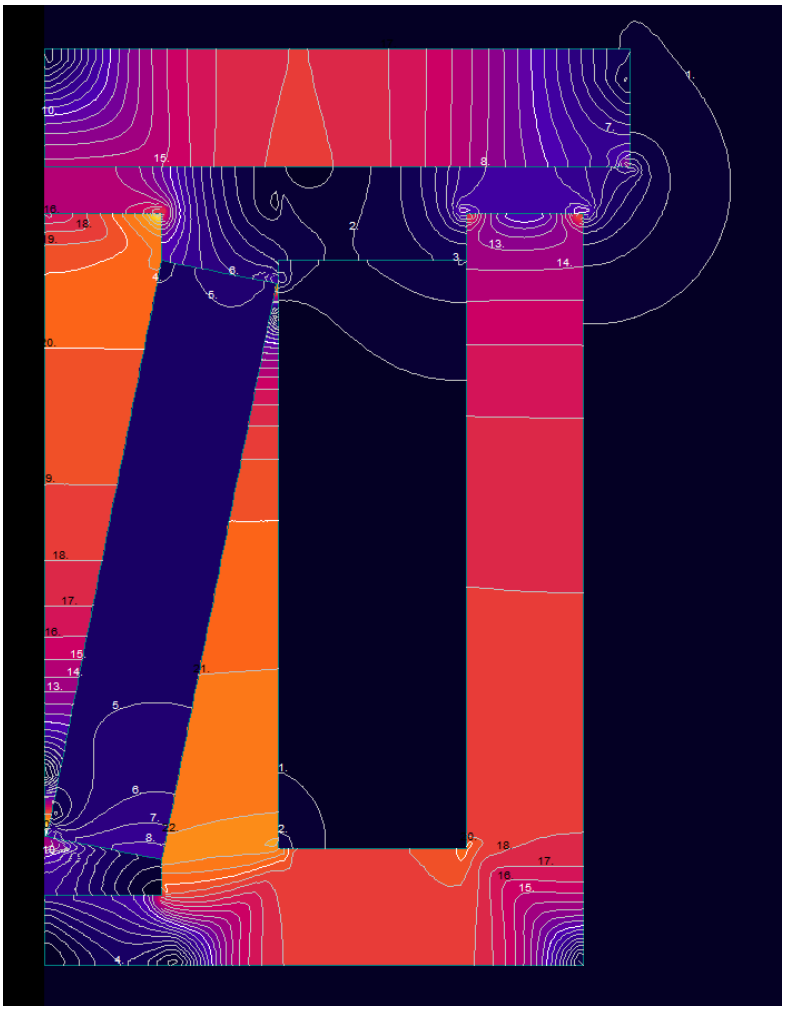

Figure 6. Simulated field map for symmetrical half of hybrid suspension system with V-shaped PM (Fig. 4). Coil currnt $I=10 \mathrm{kA}$, air gap $h=10 \mathrm{~mm}$.

\section{Conclusions}

A promising design solution is described for a hybrid PM-EM suspension system for maglev transport. The proposed configuration ensures low stray field and reduced power consumption. Established computational techniques enable generation of extensive parametric databases as well as synthesis and optimization of magnetic levitation systems for practical implementation. The analysis shows that the hybrid suspension systems have the potential of a compact design with a low power consumption and increased load capacity as compared to conventional EMS. 


\section{References}

Amoskov, V.M., Belov, A.V., Belyakov, V.A. et.al. (2008) Plasma Devices and Operations. 16(2), pp. 89103.

Amoskov, V.M., Arslanova, D.N., Bazarov, A.M. et.al. (2014) VESTNIK of St. Petersburg University. Applied mathematics. Computer science. Control processes. Ser. 10 Issue 4, pp. 5-15.

Amoskov, V.M., Arslanova, D.N., Bazarov, A.M. et.al. (2014) Problems of Atomic Science and Technology, Ser. Fusion. 37(4), pp. 84-95

Amoskov, V.M., Arslanova, D.N., Bazarov, A.M. et.al. (2015) VESTNIK of St. Petersburg University. Applied mathematics. Computer science. Control processes. Ser. 10 Issue 2, pp. 8-32.

Amoskov, V.M., Arslanova, D.N., Bazarov, A.M. et.al. (2015) VESTNIK of St. Petersburg University. Applied mathematics. Computer science. Control processes. Ser. 10 Issue 3, pp. 4-21.

Amoskov, V.M., Arslanova, D.N., Bazarov, A.M. et.al. (2016) VESTNIK of St. Petersburg University. Applied mathematics. Computer science. Control processes. Ser. 10 Issue 3, pp. 4-17.

Amoskov, V., Arslanova, D., Baranov, G., et.al. (2018) Cybernetics and physics. 7(1), pp. 11-17

Amoskov, V.M., Arslanova, D.N., Bazarov, A.M. et.al. (2018) VESTNIK of St. Petersburg University. Applied Mathematics. Computer Science. Control Processes. 14(4), pp. 286301

Amoskov, V.M., Belov, A.V., Belyakov, V.A. et.al. (2019) VESTNIK of St.Petersburg University. Ser. 15 Issue 1, pp. 5-21

Andreev, E.N, Arslanova, D.N, Akhmetzyanova, E.V., et.al. (2019) Technical physics, 89(7), pp. 1123-1129

Balandin D., Kogan M., Biryukov R. (2017) Cybernetics and physics. 6(2), pp. 57-63

Bocharov, V., Nagorsky. V. (editors) (1991) Vehicles with magnetic suspension. Mashinostroenie. Moskow.

Dzenzersky, V., Omelyanenko, V., Vasiliev, S., Matin, V., Sergeev, S. (2001) High-speed levitating transport with electrodynamic suspension. Naukova dumka.
Kiev.

Erkan, K., Okur, B., Koseki, T., and Yigit, F. (2011) IEEE International Conference on Mechatronics, pp. 23-28

Kim, K.K. (2007) Transportation systems employing magnetic suspension and superconducting magnet technology. Educational and Methodological Centre for Rail Transport Technology. Moscow.

Kim, C.-H., Cho, H.-W., Lee, J.-M., et.al. (2010), International Conference on Control, Automation and Systems (ICCAS), 27-30 Oct., 2010, pp. 732-735

Kim, K., Han, J., Kim, C., et.al., (2013) J.Electr.Eng.Technol, 8(6), pp. 1571-1578.

Morishita, M., Azukizawa, T., Kanda, S., et.al. (1989) IEEE Transactions on Vehicular Tech., 38(4), pp. 230236.

Onuki, T. and Toda, Y., (1993) IEEE Transatcions on Magnetics, 29(2), pp. 1783-1786

US patent 7533616 (2005) Guiding magnet system and magnetic levitation vehicle equipped therewith

RF patent 2573524 (2014) Combined magnetic suspension of vehicle.

RF patent 2611858 (2015) Adjustable magnetic suspension of vehicles with lifting force correction.

RF patent 2739939 (2020) Hybrid electromagnet for maglev system.

RF patent 2743753 (2020) Hybrid electromagnet without scattering fields for the maglev systems.

Safaei, F., Suratgar, A.A., Afshar, A., Mirsalim, M. (2015) IEEE Transactions on Energy Conversion, 30(3) pp.1163-1170

Tzeng, Y.-K. and Wang, T.C., (1994) IEEE Transatcions on Magnetics, 30(6), pp.4731-4733

Wang, T.C. and Tzeng, Y.-K. (1994) IEEE Transatcions on Magnetics, 30(6), pp.4734-4736

Zaitzev, A.A., Talashkin, G.N., Sokolova, Y.V. (2010) Transport utilizing magnetic suspension technology. St.Petersburg State Transport University. St.Petersburg.

Zhuravlyov, Y.N. (2003) Magnetic bearings. Theory, calculation, application. Polytechnika. St.Petersburg. 